Voix et Images

voixetimages

\title{
« Duplessis » à la télévision
}

\section{Jean Fisette}

Volume 4, numéro 2, décembre 1978

Guy Lafond

URI : https://id.erudit.org/iderudit/200160ar

DOI : https://doi.org/10.7202/200160ar

Aller au sommaire du numéro

Éditeur(s)

Les Presses de l'Université du Québec

ISSN

0318-9201 (imprimé)

1705-933X (numérique)

Découvrir la revue

Citer cet article

Fisette, J. (1978). « Duplessis » à la télévision. Voix et Images, 4(2), 319-329.

https://doi.org/10.7202/200160ar

Ce document est protégé par la loi sur le droit d'auteur. L'utilisation des services d'Érudit (y compris la reproduction) est assujettie à sa politique d'utilisation que vous pouvez consulter en ligne.

https://apropos.erudit.org/fr/usagers/politique-dutilisation/
Cet article est diffusé et préservé par Érudit.

Érudit est un consortium interuniversitaire sans but lucratif composé de l’Université de Montréal, l'Université Laval et l'Université du Québec à Montréal. Il a pour mission la promotion et la valorisation de la recherche. https://www.erudit.org/fr/ 


\section{«Duplessis» à la télévision}

La saison printanière (1978) de Radio-Canada a connu deux événements d'envergure : l'un qui vient de marquer le grand rythme annuel de la télédiffusion, soit les finales de la coupe Stanley; la régularité du cycle était d'autant mieux assurée que le club de hockey des Canadiens de Montréal non seulement a atteint les finales mais encore a-t-il remporté la coupe. Jusque-là, la périodicité, l'habituel a été assuré. D'autre part, précédant ce grand tournoi, il y eut un grain de sable dans l'engrenage, un autre événement d'envergure, spécial celui-ci, orphelin pour tout dire, qui est venu mettre en cause l'uniformité sécurisante de la programmation, soit la série: “Duplessis", présentée en février et mars dans le cadre de l'émission: Hors série. Jamais ce titre ne s'était encore réalisé de façon si manifeste. Cet écart par rapport au train-train habituel fut tel qu'il conduisit à une mise en cause publique de la fonction de la télévision dans la société. En font preuve les nombreuses et très divergentes opinions publiques, issues de toutes parts, qui ont accompagné et suivi la télédiffusion du feuilleton.

C'est qu'un point sensible avait certainement été touché, voire excité. Pour rendre compte de cet événement, de son impact et surtout de sa signification, on devra préalablement s'interroger, dans la perspective d'une sémiologie de la production télévisuelle, sur la signification de la programmation en rapport avec le diffuseur et le public récepteur. Car c'est sur le fond de scène de la programmation habituelle que l'événement "Duplessis» tire son relief, affiche sa dissidence.

\section{Le grand malaxeur}

"La télévision nous regarde ", disait J.-P. Lefebvre dans une livraison précédente de notre revue. Elle le fait si bien que la programmation, respectée d'une façon quasi maniaque (sauf pour le dieu de l'Arène, le Sport), finit par créer chez le téléspectateur des attentes imposées artificiellement, pire, une structure d'objets de désir. 
Le tableau des émissions matérialise, réalise cette orientation forcée des attentes. Ainsi, pour retenir les catégories les plus évidentes, je proposerai celles-ci :

A. Le divertissement, comprenant des niveaux culturels que l'on peut aisément hiérarchiser, du plus recherché au plus populaire: 1-Ciné-Club, les Beaux dimanches (téléthéâtre, concert de musique «sérieuse»); 2- téléroman, feuilleton, variétés musicales, cinéma de fin de soirée: 3- séries policières (presque toutes d'origine américaine), quizz, etc.

B. D'autre part, quelques émissions d'affaires publiques suivies, en rapport avec le même principe de hiérarchisation, des différents bulletins de nouvelles.

C. Enfin, celles du sport, aussi hiérarchisées, depuis les grandes finales de hockey jusqu'à la diffusion de tournois de quilles et de lutte.

Or, ce tableau que nous venons de tracer, s'il correspond à l'image que l'on se fait habituellement d'une programmation, est trompeur: qu'il y ait chez les diffuseurs volonté de diversification suivant les deux critères proposés du niveau culturel et des champs d'intérêts, il n'y a pas à en douter. Par contre, on doit éviter d'assimiler l'effet télévisuel à une sélection des spectacles donnés en ville dans les différentes salles.

Le spectacle d'une partie de hockey, par exemple, aura un impact différent selon que le spectateur y assiste au Forum, noyé dans une foule survoltée, ou qu'il en reçoive la diffusion par l'intermédiare de la télévision. La description de la partie (faite de halètements, de variations dans le débit et le ton, etc.) se substitue alors à l'atmosphère surchauffée du Forum. Si bien que les spectateurs varient fondamentalement: d'une part, une scène et un choral spontané d'admiration et de déception (c'était d'ailleurs exactement le rôle des chœurs dans la tragédie grecque), d'autre part, une "voix et une image".

Si la télévision s'approprie et refait ainsi le spectacle original, un autre postulat apparaît encore plus significatif : c'est que la télévision a une forte propension, en raison de la limitation de ses propres moyens techniques, à «similariser» les différents spectacles.

Ainsi, que je sois visé ou non, de par mon appartenance à une classe culturelle, de par mes intérêts, l'animateur d'un quizz, le surhomme du "far-west " ou la personne de Duplessis, le violoniste-soliste ou Diane Dufresne, le Pierre Nadeau du Télé-Mag ou le Mickey Mouse de Walt Disney, Aline Desjardins ou Léon-Joseph Beaulieu, tous deviennent des personnages de même dimension, tous entrent par la même porte dans mon salon, tous me sont «donnés" par le même commanditaire.

Si bien qu'il se crée une scène commune, un lieu de déplacements entre les diverses catégories d'émissions : les finales de hockey mettant en confrontation les Canadiens de Montréal et les Maple-Leaf de Toronto pren- 
nent une valeur sinon une signification bien arrêtée, sur les plans politique et historique (même les questions linguistiques y affleurent) alors qu'inversement l'entrevue accordée par un politicien se transformant, en vertu des lois inhérentes au médium, en interrogatoire devant le tribunal populaire, compte parmi les meilleurs matchs auquel il nous soit donné d'assister; là le journaliste se fait avocat-procureur du peuple télévisuel, ailleurs l'avocat se fait journaliste.

La télévision agit comme un malaxeur, produisant une sorte de pâte plus ou moins homogénéisée, UN spectacle global.

Si les journaux nous fournissent des grilles-horaires, c'est justement pour entretenir chez le téléspectateur l'illusion d'une discrimination entre les diverses productions, pour lui donner à croire que la programmation consacre des périodes d'antennes particulièrement adaptées à ses goûts. J'avais proposé plus haut l'expression : "structure d'objets de désir "; dans les effets de la télédiffusion, il n'y a plus aucun principe de distinction qui tienne, plus de structuration à proprement parler: les objets de désir prennent la forme unique d'un espace d'attente : seuil d'un lieu savamment aménagé de sensations aiguillonnées, angoisse dosée avec soin et, pour finir, "happy ending" rétablissant l'équilibre initial : tout est bien qui finit bien! Le spectateur peut aller dormir!

Ce modèle simple est d'ailleurs tellement contraignant, que seuls les quelques événements sur lesquels la machine n'a pas une prise totale, c'est-à-dire les spectacles à aboutissement imprévisible, reçoivent le privilège de déborder les cadres de la programmation, de déplacer ou effacer le déroulement normal de l'horaire: les congrès politiques, les courses au leadership, les soirées d'élection et les grandes finales sportives, bref nos deux sports nationaux.

De par leur caractère d'exception, ces retransmissions/productions viennent, par l'absurde, attester de la rigidité de la programmation de base. II y a une interdépendance très forte entre un schéma d'émission et le fait qu'elle jouisse d'une case réservée dans la grille horaire.

\section{Un potentiel diminué ?}

Dès lors, quelques questions se posent : la télévision est-elle vraiment en mesure de produire des émissions dites «d'information" ou «d'affaires publiques » qui échappent à la forme «fictionnalisée», ou «narrativisée» du feuilleton? Pendant les mois d'été, Télé-Métropole laisse ses spectateurs sur la question suivante : Léon-Joseph Beaulieu meurt-il ou bien effectuerat-il le passage au nouveau feuilleton annoncé? Durant plus d'un mois, le Téléjournal a laissé ses spectateurs sur la même question : Aldo Moro est-il mort? Mourra-t-il? Peu importent les implications: c'est une question étrangère ici; sémiologiquement, la structure formelle est la même (celle du processus de suspense) et, corrélativement, l'habitude de perception est la même. C'est la pâte plus ou moins homogénéisée dont je parlais plus haut! 
Posons le problème en d'autres termes: comment la structure télévisuelle parviendra-t-elle à discriminer vraiment divertissement, affaires publiques et télévision éducative? C'est là, entre autres, un problème que n'a pas réussi à résoudre Radio-Québec condamné ou privilégié par le C.R.T.C. à s'en tenir à la télévision éducative (je reviendra sur le sujet).

Dans quelles mesures la télévision peut-elle produire des émissions dites de "divertissement" (par exemple du quizz au feuilleton) qui échappent aux préoccupations socio-politiques, aux instances idéologiques?

On a suffisamment démontré que la production culturelle à l'allure la plus innocente est profondément marquée idéologiquement ("rien n'est innocent"; "tout a du sens ou rien n'en a») : inscrivons là une première contingence. Comme j'ai tenté de le démontrer plus haut, l'institution télévisuelle vient en ajouter une seconde, qui, elle, est formelle : soit le schéma stéréotypé de l'émission répondant au facteur d'homogénéisation.

Mais cette distinction est analytique, spéculative: en fait, les deux contingences sont liées comme le signifiant et le signifié : c'est que'le facteur d'homogénéisation n'est rien d'autre que la contre-partie nécessaire de la contingence idéologique puisqu'ils servent les mêmes intérêts, obéissent aux mêmes lois.

Ainsi, dans une perspective de la communication, on pourrait proposer ce schéma simple du médium télévisuel :

$$
\text { Source } \rightarrow \text { Message } \rightarrow \text { Récepteur }
$$

Suite aux propos tenus plus haut, je proposerai qu'une catégorisation de l'auditoire implique une classification semblable des "messages", soit la grille horaire.

Or il se produit que le diffuseur ne vend pas des produits-messages aux téléspectateurs mais qu'il vend plutôt des auditoires au publiciste ${ }^{2}$, si bien que ce premier schéma n'est pas une relation de base, mais un objet entrant dans un circuit d'échange commercial, soit :

\section{Diffuseur $\rightarrow$ Produit d'échange $\rightarrow$ Publiciste \\ Source-message-récepteur}

\section{AUDITOIRE}

Dans la mesure où le diffuseur a intérêt à "produire" un auditoire le plus large possible, il cherchera à élaborer un «message» qui soit le plus polyvalent possible face aux différentes catégories de téléspectateursrécepteurs, d'où le phénomène de mixage déjà signalé.

Ce schéma à trois paliers est donc nécessaire pour rendre compte de l'intime imbrication des deux principes de contingence. L'importance primordiale accordée aux cotes d'écoute dans le monde de la télédiffusion vient attester de la position centrale qu'occupe le terme "auditoire" dans ce schéma ${ }^{3}$. 
Terminons cette introduction théorique sur une proposition générale : une émission donné, quelle qu'elle soit, est par la force des choses tenue dans une position ambiguë d'équilibre instable entre d'une part, son appartenance à une catégorie de spectacle délimitée par des habitudes de pensée extra-télévisuelle et, d'autre part, par son médium producteur, l'institution de la télévision, qui tend à la fusionner dans l'ensemble de la production.

Cette position d'ambiguitté, que l'on peut représenter par le couple :

\section{fusion opposée à différence}

est résolue au niveau du public téléspectateur qui reçoit, décode et interprète les messages qui lui sont imposés. La seule question pertinente est alors de mesurer la marge de manœurre qui lui est laissée.

C'est en ce sens qu'une émission ne répondant pas aux critères les plus habituels (lire: les normes les plus sûres) reste toujours une bouteille lancée à la mer et constitue un lieu privilégié pour analyser les possibilités et les réalisations d'une véritable communication et réflexion télévisuelles (cette dernière expression est certes ironique). Plus encore: ces rares événements, où la différence ne vient plus équilibrer le principe de mixage, permettent de faire le procès de la télévision.

\section{"Duplessis n: une production super-télévisuelle}

Nombreuses sont les raisons qui ont pu motiver la direction des programmes de Radio-Canada à commander la série "Duplessis", tout en y investissant un budget considérable.

On se rappellera les grands succès qu'ont connus les productions récentes de la C.B.C. (réseau anglophone d'État) portant sur la crise d'Octobre et sur la situation historique et socio-politique des Canadiens français. II y a là un évident jeu de concurrence. Pourquoi alors, dira-t-on un feuilleton et non une série de dossiers?

La première raison que j'invoquerai est qu'en général, les dossiers d'affaires publiques et les dossiers d'histoire, de par les moyens techniques mis en œuvre pour les réaliser, appartiennent plus à la radio qu'à la télévision; c'est-à-dire que dans ces productions, très souvent, l'image n'est qu'un surplus, une prime, un accessoire visant à assurer un effet de concrétisation, l'image est tautologique par rapport au discours central qui est le tout : la version télévisuelle du dossier, tout comme un livre illustré, réalise à merveille cette expression populaire (toujours donnée dans les manuels de rhétorique comme un exemple du pléonasme) : “Je l'ai vu de mes yeux vu." II n'est que d'imaginer un instant ce qu'aurait pu être une série de dossiers consacrés au même sujet pour sentir aussitôt que presque tout l'impact tombe. 
Soit une série de feuilletons. On peut alors rappeler le succès tout aussi important qu'a connu la série des Rois maudits produite par l'O.R.T.F. et diffusée à deux reprises par la télévision d'État. On peut facilement imaginer que la société Radio-Canada, dans une perspective d'échange de productions télévisuelles, ait voulu, elle aussi, produire sa série prestigieuse. Dans ce cas, s'il s'agissait de produire un feuilleton apparenté au genre du "roman historique", pourquoi avoir choisi une période si récente? Probablement - comme cet événement télévisuel nous l'aura démontré parce que le duplessisme, sujet toujours tabou, est malgré tout bien vivant.

II est encore d'autres raisons, extra-télévisuelles celles-là, que l'on doit évoquer: c'est que depuis quelques années, les sujets historicopolitiques se vendent très bien dans les librairies. L'heure est aux débats de cet ordre, particulièrement dans un Québec en instance de référendum national; le sujet devait nécessairement provoquer une curiosité sinon un intérêt d'autant plus grand que la diffusion s'inscrivait dans une période de vacuum d'information en raison de la paralysie des grands journaux.

A la suite du gouvernement du Québec, la société Radio-Canada élevait, elle aussi, son monument à Duplessis et, du même coup, administrait son propre pied-de-nez au prince mécène de l'Outaouais, un antiduplessiste notoire!

Je voudrais proposer ici deux autres remarques d'ordre sémiologique, concernant la périodicité des épisodes successifs et l'aspect proprement romanesque de la production.

D'abord la périodicité est une caractéristique fondamentale de la grille-horaire; c'est par cette rythmique (dont l'unité est la semaine) que la télévision réussit cet exploit de ne jamais déranger ni surprendre, tout en imposant un espace d'attente régulièrement satisfait. Ce schéma est d'ailleurs omniprésent, que ce soit dans le téléroman où la séquence est la plus explicite parce que narrativisée (qu'arrive-t-il ? Suite la semaine prochaine, même heure, même poste!), le feuilleton, l'émission d'affaires publiques ou le hockey du samedi soir.

Cette rythmique n'est valable que si elle s'étend sur une période annuelle. Les habitudes ont le souffle long! Si bien qu'une série couvrant une période beaucoup plus restreinte prend un caractère d'exception; par exemple: les finales de hockey. Au point que l'on peut se demander si c'est le contenu ou la divergence par rapport au rythme hebdomadaire uniforme qui produit ce caractère d'exception.

D'autre part, dans une série échelonnée sur quelques semaines, la cohésion interne, les rapports entre les épisodes doivent être beaucoup plus serrés et beaucoup plus efficaces que dans la série annuelle pour suppléer à la carence de l'habitude. On imagine facilement qu'une grande partie de l'auditoire sait par cœur, sans effort et depuis longtemps, que l'émission d'affaires publiques occupe la case du mardi soir, $21 \mathrm{~h} 30$. La 
diffusion d'une série spéciale exige du spectateur une mémoire spécifique et des changements d'habitude. L'obtention de cet effort, ça se gagne...

Par tous ces aspects, la série «Duplessis» se doit d'être rapprochée des grandes finales de Hockey à la différence près que ces séries sportives sont, elles, inscrites dans le grand rythme annuel de la programmation télévisuelle.

De tous les commentaires qui ont entouré la diffusion de cette série, je n'en ai rencontré qu'un seul qui soit vraiment intéressant. Dans une analyse pénétrante, Jean-Pierre Desaulniers ${ }^{4}$ procédait à une comparaison entre ce personnage de Duplessis et cet autre fantôme qui habite notre radio-télévision depuis 25 ans, Séraphin Poudrier : même goût pour un pouvoir épuré des jouissances matérielles qu'il permet, même sexisme qui renvoie la femme dans l'ombre de la servilité, mêmes contacts personnels avec les "sujjets", même système de faveurs qui permet de constituer des dossiers de mémoire et ainsi d'assurer une poursuite du pouvoir fondé sur la crainte ou le chantage, même sobriété, ici gagnée, là acquise préalablement, mêmes interdits sexuels, même crainte de Dieu se manifestant par une foi naīve, mais sincère, mêmes rapports ambigus d'admiration mais d'éloignement prudent face au clergé... Les lieux de similitude sont étonnamment nombreux, au point que l'on peut se demander si le scénariste de la série «Duplessis» ne s'est pas inspiré, consciemment ou non, du grand radio-roman des années cinquante pour asseoir la vraisemblance et reproduire la psychologie de l'époque. Ou bien, à l'inverse, se demanderat-on si le radio-roman Un homme et son péché n'avait pas comme fonction, durant les années cinquante de refléter, de donner comme normatif et finalement, comme allant de soi, une image très spécifique du pouvoir. (II y a là une hypothèse qu'il faudrait pousser plus loin un jour : le Premier ministre Robert Bourassa n'a-t-il pas eu son double télévisuel dans la figure de ce prêtre-ouvrier de la Rue des pignons, refuge des âmes en peine, non pas contestataire pour un sou, mais fébrile travailleur social, professionnel des cataplasmes; et le Premier ministre René Lévesque ne trouve-t-il pas son double dans ce Grand-papa ouvert, compréhensif mais peu loquace, renvoyant ses enfants à leur sens de l'honneur - et de la famille - puis à leurs responsabilités, n'intervenant que pour éviter le pire: une sorte de garde-fou à distance?)

Mais alors pourquoi, en 1978, ce retour en force du personnage de Séraphin Poudrier? La couleur historique est insuffisante pour tout expliquer. Et si cette structure du pouvoir était restée imprégnée dans l'imaginaire des Québécois? Chose certaine, c'est que le succès, encore vivace aujourd'hui, des Belles Histoires des pays d'en haut (ce titre s'appliquerait fort bien à la série) a certainement contribué pour beaucoup dans la fascination qu'a exercée la série: un succès qui, dans cette perspective, serait à mettre au compte d'un espace d'attente bien fondé et qui a été généreusement comblé. 
Cette brève revue de quelques aspects de la série «Duplessis " vient illustrer le principe d'homogénéisation proposé plus haut : de quelque côté qu'on l'examine, il est impossible de confiner cette série dans une catégorie spécifique : affaires publiques, télévision éducative, feuilleton, compétition, reconstitution d'époque; il faut faire appel à tous ces traits pour en rendre compte avec un minimum de justesse.

$\mathrm{Si}$, comme on a tenté de le démontrer plus haut, toutes les émissions produites par et pour la télévision ont une forte tendance à se fusionner dans un spectacle unique, la série «Duplessis" a réalisé, avec excès, ce mixage. A ce point, d'ailleurs, que les téléspectateurs en ont été profondément dérangés dans leurs habitudes. S'il y a eu sentiment de différence, ce ne fut qu'en regard de la programmation institutionnelle. D'où la situation exceptionnelle et l'indice de divergence.

\section{L'apprenti-sorcier}

Une production qui réalise avec excès les virtualités propres au médium télévisuel et un public télévisuel qui n'en finit plus de protester : voilà le paradoxe!

Je ne reprendrai pas ici toutes les critiques, protestations et dénonciations qui ont fourmillé à la suite de la diffusion de la série : il serait d'ailleurs impossible de les résumer tant elles sont divergentes. Les thèmes entendus le plus fréquemment référaient à l'exactitude historique et surtout au manque de respect dans le traitement des personnages: niveaux de langage, gestes familiers, tendance à l'alcoolisme, etc... Le public plus âgé eut l'impression que son idole avait été bafouée.

(Chose curieuse, une brève enquête auprès des jeunes, qui n'avaient pas connu le régime Duplessis a révélé qu'à leurs yeux, le personnage avait grandi.)

Ce ton de familiarité n'est au fond rien d'autre que le déplacement d'un sujet du type dossier historico-politique vers le traitement romanesque du feuilleton: c'est le médium télévisuel qui ici impose ses propres lois, informe un contenu. Ce n'est d'ailleurs pas un hasard que cet auditoire des plus jeunes, formés tôt à la télévision, ait tiré une image positive du personnage.

Une autre critique concernait la similarité, très évidente à certains moments, des propos, slogans et discours politiques tenus par les personnages de la série et les représentants du gouvernement actuellement au pouvoir à Québec. Le même facteur d'homogénéisation vient encore jouer ici entre les "Affaires publiques", plus précisément la campagne électorale, et le feuilleton.

Soulignons simplement, pour terminer, ces rencontres Duplessiscardinal Villeneuve qui reproduisent autant un match sportif, une assemblée 
contradictoire qu'une entrevue un peu serrée... M. J. Lacourcière, l'historien qui a assumé la recherche fondamentale, a eu beau arguer que toutes ces répliques figuraient dans la correspondance échangée entre les deux personnages, on continue à protester, affirmant que de telles rencontres n'avaient jamais eu lieu.

II serait possible de continuer à gloser ainsi sur ces critiques qui renvoient inévitablement toutes à cette caractéristique télévisuelle du mixage des genres.

Le scénario a été construit de façon à intégrer, de façon étonnante, ces types de discours fort différents; il faut voir que seul cet ingénieux mixage permettait de rendre compte des aspects si divers du personnage: la dureté de l'homme qui commandait de réprimer sauvagement les manifestations ouvrières, mais la douceur toute paternelle de celui qui faisait découvrir le nouveau grille-pain automatique à un enfant; le jeune tribun qui dénonçait le népotisme mais aussi le «grand patronneux" du Québec: candidat défait, isolé dans sa chambre craignant d'affronter le peuple, mais le chef fier donnant un drapeau à la province; le politicien à l'écoute attentive de son informatrice anonyme, mais aussi le chef qui donne des leçons de rhétorique à ses ministres: "On ne dit pas "dépenser", mais «consacrer» un budget. »

Si l'emprunt aux diverses catégories du discours télévisuel était intimement lié à la globalité du portrait de l'ère duplessiste, provoquant, par le fait même, une multitude de protestations, on peut inférer que ce qui a été mis en cause, c'est le médium télévisuel lui-même. En d'autres termes, on faisait le procès de la télévision.

Ou plutôt, l'auditeur se sentait écrasé, impuissant devant la réalisation: on a suggéré plus haut que la seule question vraiment pertinente serait de mesurer la marge de manœuvre qui lui est laissée dans son travail de décodage-interprétation.

Or il s'est produit, dans ce cas particulier, que cette marge de manœuvre était pratiquement nulle : la composition du scénario était tellement serrée, l'image de l'époque duplessiste était tellement polyvalente que la production ne pouvait que parcelliser l'auditoire, renvoyer les téléspectateurs dos à dos, chacun dans l'insignifiance de sa petite critique, impuissant devant la globalité et la perfection de la machine.

En ce sens, il serait plus juste d'affirmer que c'est la télévision qui a institué son propre procès 5 .

Et je reprendrai ici une des questions que j'ai posées plus haut : dans quelles mesures le médium télévisuel peut-il produire une émission qui remplisse vraiment une fonction sur la place publique ${ }^{6}$, c'est-à-dire de lancer un débat, d'ouvrir la discussion, de rendre une société consciente de sa propre configuration, de faire progresser la connaissance qu'a le citoyen de ses racines, de ses fantasmes, des lois régissant le groupe social auquel 
il appartient? La réponse s'est révélée nulle! La télévision, avait-on claironné lors de son apparition, serait un grand facteur d'éducation populaire, de cohésion sociale. (Et je ne doute pas que Radio-Canada ait partiellement rempli ce rôle durant ses premières années.) Et si les techniques s'étant raffinées, stéréotypées, le public s'étant habitué, la télévision était devenu un facteur d'uniformisation et de prolétarisation culturelle?

Le spectateur, en somme, a protesté contre la perte de l'uniformité sécurisante de la programmation établie, contre la perte des chères habitudes, du lien qui tient son imaginaire dans les limites étroites. Ces protestations ont été tellement dérisoires (mais au fond si tristes) qu'elles confinaient à un niveau de ridicule qu'on n'aurait su imaginer: le politicientémoin qui regrette qu'on n'ait pas tenu compte des conditions atmosphériques, l'archevêque qui, très officiellement, proteste contre le traitement quelque peu caricatural il est vrai, fait à son prédécesseur, l'écrivain de l'époque, en mal de publicité, qui cherche à profiter de l'occasion pour redorer son blason fort ombragé par les temps qui courent...

Le dernier mot reste encore aux promoteurs du projet : renoncerontils à télédiffuser de nouveau cette série? Ou bien auront-ils le courage de poursuivre dans cette voie de dissidence?

$\mathrm{Si}$, en hauts lieux, on considère l'aventure comme un désastre (l'apprenti-sorcier en a déjà connu de similaires), on se réorientera vraisemblablement vers des sujets moins explosifs : dans ce cas, je suggérerais des séries sur les gouverneurs de la Nouvelle-France ou sur Madeleine de Verchères. Alors l'auditoire redeviendra une grande famille télévisuelle, retrouvera son unanimité perdue, se divertira de nouveau et entamera en chœur avec complaisance, mais sans risques cette fois-ci : «Je me souviens!"

Jean Fisette

Université du Québec à Montréal

1. Jean-Pierre Lefebvre, «Télévision", dans Voix et images, vol. I, $n^{\circ} 3$, avril 1976 , p. 445-447.

2. Voir à ce propos: "L'industrie des média ou le marché des auditeurs", dans Recherches sociagraphiques, vol. XII, $n^{\circ} 1$, janvier-avril 1971; ce texte est reproduit dans: Communication de masse et consommation de masse, ouvrage collectif sous la direction de Claude Cossette, Boréal-Express, Sillery, Québec, 1975, p. 227-232.

3. Précisons néanmoins que la télévision d'État, jouissant d'une seule source de revenus, gouvernementale (Radio-Québec) ou d'une double source de revenus (Radio-Canada, C.B.C.) peut se permettre une certaine distanciation face aux cotes d'écoute : c'est que, dans ces cas, l'auditoire peut plus facilement être parcellisé.

4. Jean-Pierre Desaulniers, "l'Éclairage cathodique de la grande noirceur", le Devoir, le samedi 1er avril 1978. 
5. Peu avant la fin de la diffusion de la série, Radio-Canada nous a donné une prime sous forme d'un forum d'une heure consacré à la série: l'émission réunissait d'une part quelques témoins de l'époque (accusation) et d'autre part, l'historien J. Lacourcière à la défense de la production, appuyé en cela par ce célèbre ex-écrivain qui, la semaine suivante, lors de la diffusion de la dernière émission, devait se voir glorifier par Duplessis lui-même. On a alors eu droit à la caricature de ce procés de la télévision dont nous parlons. Serait-ce que les habitudes télévisuelles sont tellement imprégnées que ce procès n'a pu se faire sur la place publique? On devra en conclure que la télévision, encore ici, a réussi à récupérer la situation précaire où elle s'était mise et à en faire un spectacle de divertissement,

6. Une question qu'ont commencé à poser Jacques Godbout et Florient Sauvageau dans leur excellent film documentaire: Derrière l'image, produit par I'O.N.F. Ce film, diffusé sur les ondes de Radio-Canada en avril dernier, sera vraisemblablement donné en reprise l'automne prochain. 